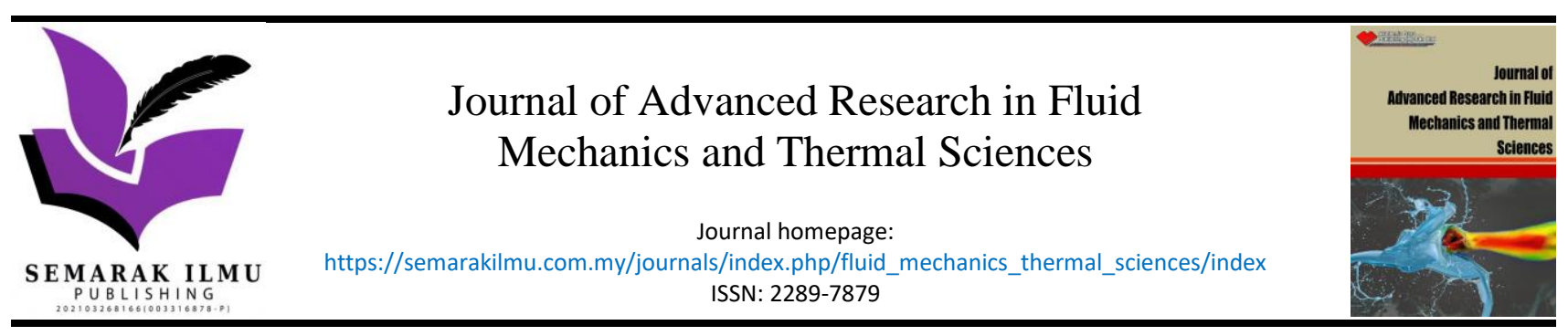

\title{
Combined Convective Transport of Brinkman-viscoelastic Fluid Across Horizontal Circular Cylinder with Convective Boundary Condition
}

\author{
Siti Farah Haryatie Mohd Kanafiah ${ }^{1,2, *}$, Abdul Rahman Mohd Kasim ${ }^{2, *}$, Syazwani Mohd Zokri ${ }^{3}$, \\ Mohd Rijal Ilias ${ }^{4}$ \\ Universiti Teknologi MARA, 18500 Machang, Kelantan, Malaysia \\ Universiti Malaysia Pahang, 26300 Kuantan, Pahang, Malaysia \\ Universiti Teknologi MARA, 21080 Kuala Terengganu, Terengganu, Malaysia \\ Faculty of Computer and Mathematical Sciences, Universiti Teknologi MARA (UiTM), 40450 Shah Alam, Selangor, Malaysia
}

\section{ARTICLE INFO}

Article history:

Received 5 August 2021

Received in revised form 25 October 2021

Accepted 5 November 2021

Available online 29 November 2021

\section{Keywords:}

Combined convective transport; horizontal circular cylinder; convective boundary condition; porous medium

\section{ABSTRACT}

Traditional heat transfer fluids frequently encounter several limitations in the heat transfer process, due to the lower thermal conductivity in heat transfer process industries, and also has an impact on the performance of heat transfer in industrial sectors. In order to overcome the problem, researchers have currently considered an alternative development of heat transfer of fluids. Hence, this study will concentrate on the problem of steady combined convective transport. In particular, the flow of Brinkman-viscoelastic fluid over a horizontal circular cylinder with the influence of convective boundary condition $(C B C)$ was investigated. Using the necessary similarity transformation, the governing equations were converted into a less complicated form and numerically solved by using Runge-Kutta-Fehlberg-method, which was programmed in Maple software. The influence of Biot number, combined convection, Brinkman and viscoelastic parameters are analyzed and demonstrated in graphs and tables. Numerical result showed that the fluid velocity increased with improving conjugate and combined convection parameter, but decreased with increasing Brinkman and viscoelastic parameter. It is also discovered the reverse trend on temperature profiles.

\section{Introduction}

Heat transfer plays a vital role in real world applications throughout the fluid flow. The fluid temperature can influence the fluid flow characteristic, particularly when the flux has a buoyancy force. Convection is the most common case in fluids among the types of heat transfer. Because of potential applications in industries such as oil exploration, construction equipment, cosmetic products, and blood flow, the research on convective heat transfer has received special attention. Combined convective transport is one of the types of convection and also known as situations where

\footnotetext{
* Corresponding author.

E-mail address: sitif315@uitm.edu.my

* Corresponding author.

E-mail address: rahmanmohd@ump.edu.my
}

https://doi.org/10.37934/arfmts.89.2.1524 
both the forces of pressure and the forces of buoyancy interact. Recently, there have been extensive interest on free as well as combined convective transport past a horizontal circular cylinder [1-7].

The investigations on non-Newtonian type fluid have gained more attention by researchers in past few decades since the conventional fluids (Newtonian) are said to be unfitting to represent the fluid that happened in industrial applications. Available investigation on non-Newtonian fluid can be found in Zokri et al., [8], Kasim et al., [9], Kasim et al., [10] and Arifin et al., [11]. Among all, Brinkman fluid is one of the models that applicable for high porosity incompressible fluid flow. Tham and Nazar [12] investigated the combined convection over a solid sphere in a porous medium immersed with nanofluid using the Brinkman model. Under the similar problem, Tham et al., [13] considered the flow across horizontal circular cylinder. They disclosed that increase in the convection parameter delayed the separation of the boundary layer.

Ali et al., [14] investigated the performance of chemical reactions on Brinkman fluid flow. They investigated how heat and mass diffusion interact with time fractional over an oscillating plate. Siyal et al., [15] studied the time changes of heat flow on the magnetohydrodynamic Brinkman fluid for the oscillations heated plate. They discovered that the fractional Brinkman fluid has an increasing temperature with an increment in fractional parameter. Islam et al., [16] considered a Brinkman fluid over an infinite plate utilising the Fourier Transformation method. Moreover, Kausar et al., [17] focused on the Brinkman flow with frictional heating and porous dissipation over a stretching sheet. Recently, the onset of convection for Darcy-Brinkman fluid was investigated by Yadav [18]. They disclosed that Darcy number and gravitational forces were delayed at the start of convection.

Furthermore, viscoelastic fluid is the most common fluid dealing with viscosity and elasticity properties. Aziz et al., [19] examined the impact of aligned magnetohydrodynamics on viscoelastic fluid with Newtonian heating over a circular cylinder. A few years later, due to similar problem, Aziz et al., [20] provided the development of a viscoelastic micropolar model. Moreover, Mishra et al., [21] probed the viscoelastic fluid with magnetohydrodynamic effect via porous medium and revealed that the magnetic field produced low concentration in higher density. Mahat et al., [22] considered the viscoelastic nanofluid model through a horizontal circular cylinder under the effect of viscous dissipation. Authors reported that the Eckert number is to increase the skin friction and decline the heat transfer coefficient. Later, Mahat et al., [23] continued the study on the heat generation and flow of thermal performance and observed that as the heat generation parameter increases, so do the heat transfer coefficient and thermal boundary layer. The report on viscoelastic fluid also can be found in Wahid et al., [24].

Another part of the boundary layer flow interest is their variation in thermal boundary condition. The convective boundary condition $(\mathrm{CBC})$ is the most common boundary condition in practise, as most heat-transfer surfaces at certain parameters are exposed to a thermal convection environment [25]. There are many researchers in the literature considered the effect of CBC [26-29].

Inspiring by the preceding works, the combined convective transport of Brinkman viscoelastic fluid moving across a horizontal circular cylinder saturated in porous region will be included in details under thermal conditions of convective boundary condition. The Runge-Kutta-Fehlberg-Method is utilized in solving a nonlinear system. The study will focus on the variations of pertinent parameters such as Biot number, combined convection, viscoelastic, and Brinkman parameters over velocity and temperature distribution as well.

\section{Mathematical Formulation}

The steady combined convective transport flow of Brinkman-viscoelastic fluid from a circular horizontal cylinder immersed in a porous region with the effect of Biot number, is studied as 
portrayed in Figure 1 . The free stream velocity, $\frac{1}{2} U_{\infty}$ is moving up vertically through the cylinder where $T_{\infty}$ is ambient temperature which remains unchanged. The convection heats up the surface of the cylinder at temperature, $T_{f}$ that result in a heat transfer coefficient, $h_{f}$. The acceleration of gravity, $g$ acting downwards. The $\bar{x}$ coordinate is evaluated along the cylinder circumference and $\bar{y}$ coordinate is normal to the surface with a referring the radius of the cylinder.

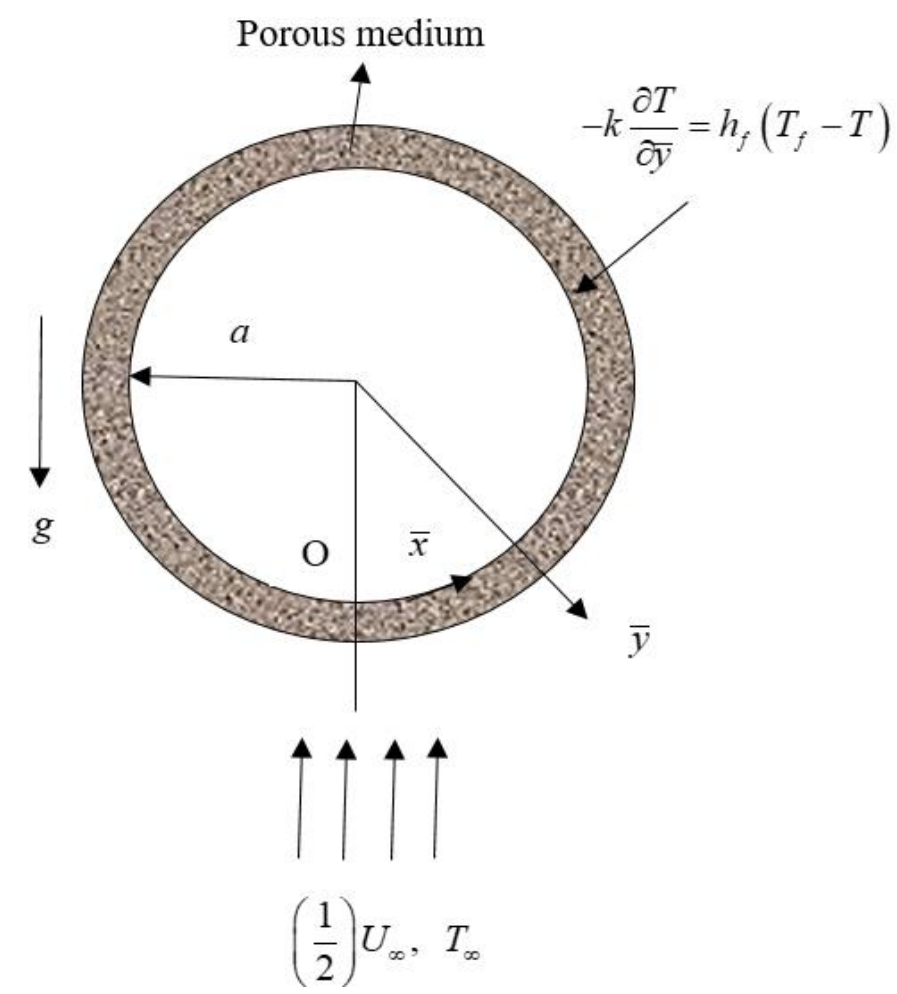

Fig. 1. Physical model of the flow

The governing equations for continuity, momentum and energy under the boundary layer approximation are as follows:

$$
\begin{aligned}
& \frac{\partial \bar{u}}{\partial \bar{x}}+\frac{\partial \bar{v}}{\partial \bar{y}}=0 \\
& \frac{\mu}{K} \bar{u}=-\frac{\partial p}{\partial \bar{x}}+\frac{\mu}{\phi}\left(\frac{\partial^{2} \bar{u}}{\partial \bar{x}^{2}}+\frac{\partial^{2} \bar{u}}{\partial \bar{y}^{2}}\right) \\
& +k_{0}\left[\begin{array}{l}
\left.\bar{u}\left(\frac{\partial^{3} \bar{u}}{\partial \bar{x}^{3}}+\frac{\partial^{3} \bar{u}}{\partial \bar{x} \partial \bar{y}^{2}}\right)+\bar{v}\left(\frac{\partial^{3} \bar{u}}{\partial \bar{x}^{2} \partial \bar{y}}+\frac{\partial^{3} \bar{u}}{\partial \bar{y}^{3}}\right)-\frac{\partial \bar{u}}{\partial \bar{y}}\left(\frac{\partial^{2} \bar{u}}{\partial \bar{x} \partial \bar{y}}+\frac{\partial^{2} \bar{v}}{\partial \bar{x}^{2}}\right)\right]-\rho g \sin (\bar{x} / a), \\
+\frac{\partial \bar{u}}{\partial \bar{x}}\left(3 \frac{\partial^{2} \bar{v}}{\partial \bar{x} \partial \bar{y}}+\frac{\partial^{2} \bar{u}}{\partial \bar{y}^{2}}\right)-2 \frac{\partial \bar{v}}{\partial \bar{x}} \frac{\partial^{2} \bar{u}}{\partial \bar{x} \partial \bar{y}}
\end{array}\right] \\
& \bar{u} \frac{\partial T}{\partial \bar{x}}+\bar{v} \frac{\partial T}{\partial \bar{y}}=\alpha_{m}\left(\frac{\partial^{2} T}{\partial \bar{x}^{2}}+\frac{\partial^{2} T}{\partial \bar{y}^{2}}\right) .
\end{aligned}
$$


depending on the boundary conditions,

$\bar{v}=0, \quad \bar{u}=0, \quad-k \frac{\partial T}{\partial \bar{y}}=h_{f}\left(T_{f}-T\right)$, at $\bar{y}=0$,

$\bar{u} \rightarrow \bar{u}_{e}(\bar{x}), \quad T \rightarrow T_{\infty}$ as $\bar{y} \rightarrow \infty$.

Where $\rho=\rho_{\infty}\left[1-\beta\left(T-T_{\infty}\right)\right]$. The velocity elements along $\bar{x}$ and $\bar{y}$ axes are referred as $\bar{u}$ and $\bar{v}$ respectively, $\mu$ is the dynamic viscosity, $K$ is porous medium permeability, $\phi$ is porosity of porous medium, $k_{0}$ is viscoelasticity, $\rho$ is fluid density, $p$ is pressure, $\beta$ is thermal expansion coefficient, $T$ is fluid temperature, $\alpha_{m}$ is effective thermal diffusivity of porous, $k$ is thermal conductivity, $h_{f}$ is heat transfer coefficient and $T_{f}$ is hot fluid temperature. The external velocity is referred to as $\bar{u}_{e}(\bar{x})=U_{\infty} \sin (\bar{x} / a)$. Eq. (1) to Eq. (4) are converted into dimensionless forms using the nondimensional variables as below:

$$
\begin{aligned}
& x=\bar{x} / a, \quad y=P e^{1 / 2}(\bar{y} / a), \quad u=\bar{u} / U_{\infty}, \quad v=P e^{1 / 2}\left(\bar{v} / U_{\infty}\right) \\
& \theta=\left(T-T_{\infty}\right) /\left(T_{f}-T_{\infty}\right), \quad u_{e}(\bar{x})=\bar{u}_{e}(\bar{x}) / U_{\infty},
\end{aligned}
$$

in which, $P e=U_{\infty} a / \alpha_{m}$ is the modified Péclet number for porous region. Subsequently, by substituting Eq. (5) into Eq. (1) to Eq. (4), the dimensionless equation can be defined as follows:

$$
\frac{\partial u}{\partial x}+\frac{\partial v}{\partial y}=0
$$

$$
\frac{\partial u}{\partial y}=\Gamma \frac{\partial^{3} u}{\partial y^{3}}+k_{1}\left[\begin{array}{l}
u \frac{\partial^{4} u}{\partial x \partial y^{3}}+\frac{\partial^{3} u}{\partial x \partial y^{2}} \frac{\partial u}{\partial y}+v \frac{\partial^{4} u}{\partial y^{4}}+\frac{\partial^{3} u}{\partial y^{3}} \frac{\partial v}{\partial y} \\
-\frac{\partial u}{\partial y} \frac{\partial^{3} u}{\partial x \partial y^{2}}-\frac{\partial^{2} u}{\partial x \partial y} \frac{\partial^{2} u}{\partial y^{2}} \\
+\frac{\partial u}{\partial x} \frac{\partial^{3} u}{\partial y^{3}}+\frac{\partial^{2} u}{\partial y^{2}} \frac{\partial^{2} u}{\partial x \partial y}
\end{array}\right]+\lambda \frac{\partial \theta}{\partial y} \sin x
$$

$u \frac{\partial \theta}{\partial x}+v \frac{\partial \theta}{\partial y}=\frac{\partial^{2} \theta}{\partial y^{2}}$

with the transformed boundary condition

$$
\begin{array}{ll}
u=0, \quad v=0, \frac{\partial \theta}{\partial y}=-B i(1-\theta) & \text { at } \bar{y}=0, \\
u \rightarrow u_{e}, v \rightarrow 0, \theta \rightarrow 0 & \text { as } \bar{y} \rightarrow \infty,
\end{array}
$$

Next, applying the similarity transformation variable: 
$\psi=x f(x, y), \quad \theta=\theta(x, y), \quad u=\frac{\partial \psi}{\partial y}, \quad v=-\frac{\partial \psi}{\partial x}$

in which $\psi$ is the stream function, while $\theta$ indicates the fluid temperature. Thus Eq. (6) is automatically fulfilled and Eq. (7) to Eq. (9) lead to:

$$
\frac{\partial f}{\partial y}=\Gamma \frac{\partial^{3} f}{\partial y^{3}}+k_{1}\left[\begin{array}{l}
x \frac{\partial f}{\partial y} \frac{\partial^{4} f}{\partial x \partial y^{3}}+\frac{\partial f}{\partial y} \frac{\partial^{3} f}{\partial y^{3}}-x \frac{\partial f}{\partial x} \frac{\partial^{4} f}{\partial y^{4}} \\
-f \frac{\partial^{4} f}{\partial y^{4}}-x \frac{\partial^{2} f}{\partial y^{2}} \frac{\partial^{3} f}{\partial x \partial y^{2}}-\frac{\partial^{2} f}{\partial y^{2}} \frac{\partial^{2} f}{\partial y^{2}} \\
+x \frac{\partial^{2} f}{\partial x \partial y} \frac{\partial^{3} f}{\partial y^{3}}+\frac{\partial f}{\partial y} \frac{\partial^{3} f}{\partial y^{3}}
\end{array}\right]+(1+\lambda \theta) \frac{\sin x}{x}
$$

$\frac{\partial^{2} \theta}{\partial y^{2}}+f \frac{\partial \theta}{\partial y}=x\left(\frac{\partial f}{\partial y} \frac{\partial \theta}{\partial x}-\frac{\partial f}{\partial x} \frac{\partial \theta}{\partial y}\right)$

subjected to boundary condition

$$
\begin{aligned}
& f=0, \quad \frac{\partial f}{\partial y}=0, \quad \frac{\partial \theta}{\partial y}=-B i(1-\theta), \text { at } y=0 \\
& \frac{\partial f}{\partial y} \rightarrow \frac{\sin x}{x}, \quad \frac{\partial^{2} f}{\partial y^{2}} \rightarrow 0, \theta \rightarrow 0 \quad \text { as } y \rightarrow \infty
\end{aligned}
$$

By setting $x \approx 0$ at the lower cylinder stagnation point, Eq. (11) to Eq. (13) are converted to a solvable system as shown below:

$$
\begin{aligned}
& f^{\prime}-\left\lceil f^{\prime \prime \prime}-k_{1}\left[2 f^{\prime} f^{\prime \prime \prime}-f f^{(i v)}-\left(f^{\prime \prime}\right)^{2}\right]-1-\lambda \theta=0,\right. \\
& \theta^{\prime \prime}+f \theta^{\prime}=0
\end{aligned}
$$

corresponding to boundary conditions

$$
\begin{aligned}
& f(0)=0, \quad f^{\prime}(0)=0, \quad \theta^{\prime}(0)=-B i(1-\theta(0)) \\
& f^{\prime}(\infty) \rightarrow 1, \quad f^{\prime \prime}(\infty) \rightarrow 0, \quad \theta(\infty) \rightarrow 0
\end{aligned}
$$

Here, (') is corresponding to the derivative with respect to $y$ and $B i$ is the Biot number. Subsequently, the dimensionless parameters in Eq. (14) are defined as in Table 1 below. 
Table 1

Definitions of dimensionless parameters

\begin{tabular}{lll}
\hline Dimensionless Parameters & Notations & Definitions \\
\hline Brinkman parameter & $\Gamma$ & $\frac{D a}{\phi} P e$ \\
Darcy number & $D a$ & $\frac{K}{a^{2}}$ \\
Mixed convection parameter & $\lambda$ & $\frac{R a}{P e}$ \\
Rayleigh number & $R a$ & $\frac{g K \beta\left(T_{w}-T_{\infty}\right) a}{\alpha_{m} v}$ \\
Viscoelastic parameter & $k_{1}$ & $\frac{k_{0} K U_{\infty} P e}{\mu a^{3}}$ \\
\hline
\end{tabular}

\section{Results and Discussion}

The numerical calculations on system of Eq. (14) to Eq. (16) were solved using the Runge-KuttaFehlberg-method, that is programmed in Maple software. The analysis is presented to examine the influence of combined convection parameter $\lambda$, Brinkman parameter $\Gamma$, viscoelastic parameter $k_{1}$ and Biot number $B i$. The assisting flow, which is $\lambda>0$ and viscoelastic fluid $k_{1}>0$ are considered in this study. According to Tham et al., [13], a large value of the Brinkman parameter implies the dominance of the no-slip condition which is limited to the viscous layer only. To characterize the Brinkman factor, $\Gamma \neq 0$ is applied. The present results were implemented by choosing the boundary layer thickness between 5 and 7 to achieve the boundary conditions asymptotically.

Table 2 shows the comparison values for the current results and Nazar et al., (2003) for verification purposes. It should be noted that when a higher value of $\mathrm{Bi}$ is used for the boundary conditions, the problems are reduced to constant wall temperature. The current results show significant agreement with existing publication, confirming the precision of the current output.

\section{Table 2}

Variations of $f^{\prime \prime}(0)$ and $-\theta^{\prime}(0)$ with $\Gamma=0.1, k_{1}=0.0001, B i \rightarrow \infty$ and various $\lambda$

\begin{tabular}{lllll}
\hline$\lambda$ & \multicolumn{3}{c}{ Nazar et al., [30] } & \multicolumn{3}{c}{ Current } \\
\cline { 2 - 5 } & $f^{\prime \prime}(0)$ & $-\theta^{\prime}(0)$ & $f^{\prime \prime}(0)$ & $-\theta^{\prime}(0)$ \\
\hline 0.5 & 4.3999 & 0.7240 & 4.3955 & 0.7237 \\
1 & 5.5923 & 0.7791 & 5.5853 & 0.7788 \\
2 & 7.8768 & 0.8706 & 7.8634 & 0.8701 \\
3 & 10.0613 & 0.9460 & 10.0400 & 0.9452 \\
\hline
\end{tabular}

Figure 2 to Figure 5 show the influence of various parameter on the velocity $f^{\prime}(\eta)$ and temperature distribution $\theta(\eta)$. The effect of various Brinkman parameter $\Gamma$ are depicted in Figure 2. The fluid velocity is found to be decreased as the value of $\Gamma$ increases due to drag force and density ratio. On the other hand, an increase in $\Gamma$ has increased the temperature distribution. Figure 3 displays the impact of combined convection $\lambda$ on $f^{\prime}(\eta)$ and $\theta(\eta)$. The fluid velocity increases with the boundary layer flow as $\lambda$ increases due to favourable buoyancy effects. Oppositely, the temperature profile decreases as $\lambda$ increases. It should be noted that the convection of heat transport is reduced and thickening the heat boundary layer. Figure 4 portrays how the fluid velocity 
and temperature increased as Biot number $B i$ increases. It is noted that the heat transfer from hot to cold side of the cylinder increases as $B i$ increases. The impact of viscoelastic parameter $k_{1}$ on the fluid temperature and velocity are displayed in Figure 5 . The velocity profile is found to be decreased for higher values of $k_{1}$. It is seen that the viscosity of fluid has slowed the velocity of fluid. Furthermore, the increase of $k_{1}$ shows the contrary trend for fluid temperature.
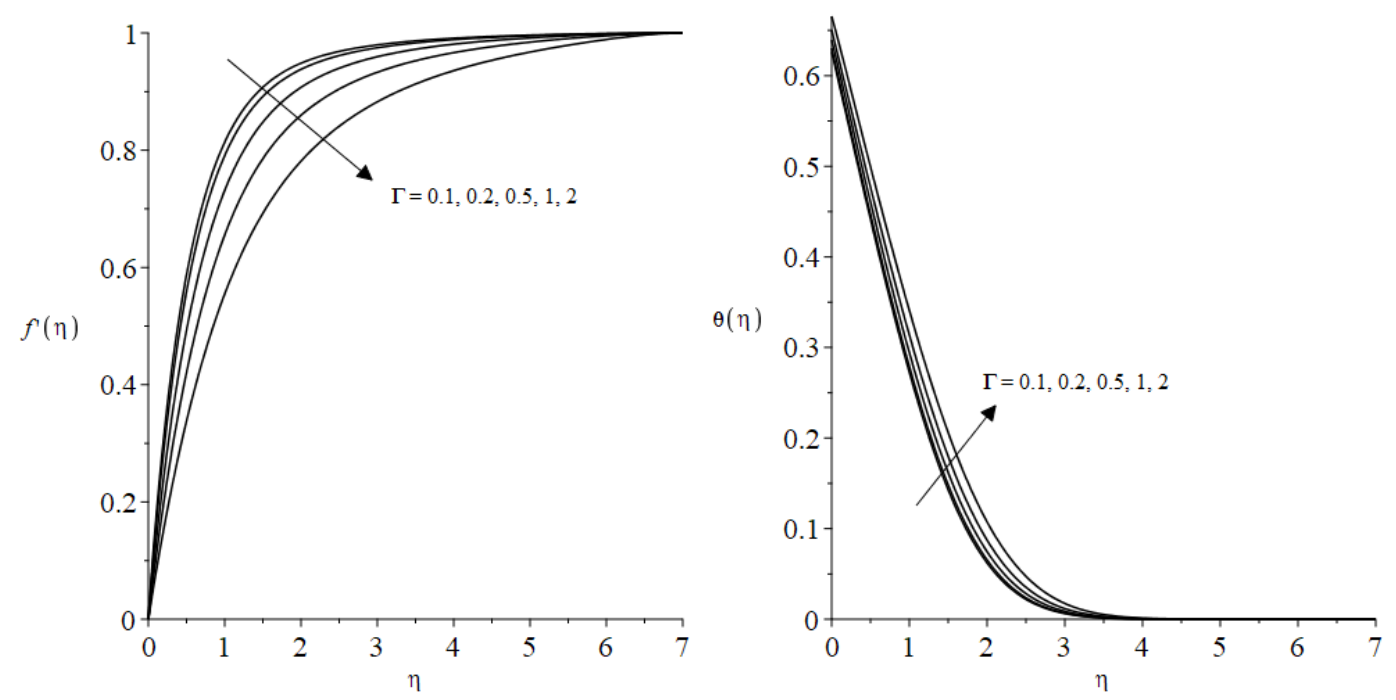

Fig. 2. Effect of $\Gamma$ on $f^{\prime}(\eta)$ and $\theta(\eta)$ when $\lambda=1, k_{1}=1$ and $B i=1$
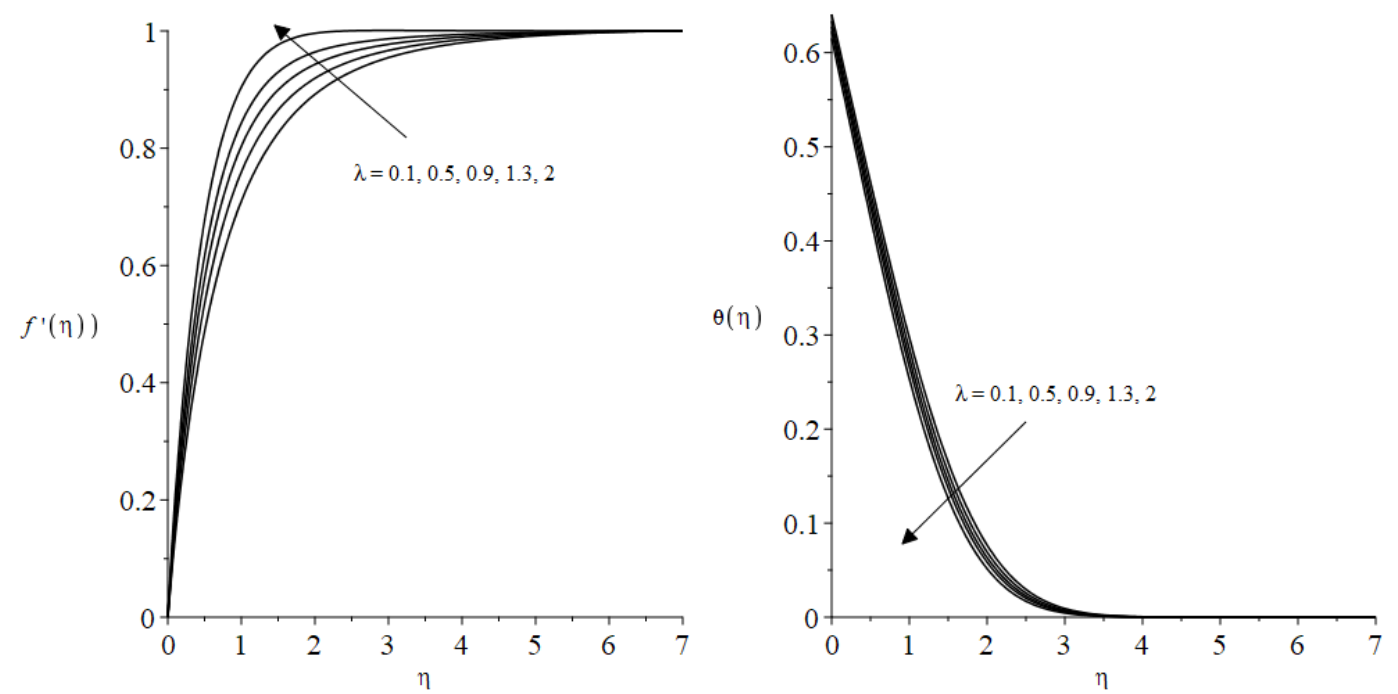

Fig. 3. Effect of $\lambda$ on $f^{\prime}(\eta)$ and $\theta(\eta)$ when $\Gamma=0.1, k_{1}=0.3$ and $B i=1$ 

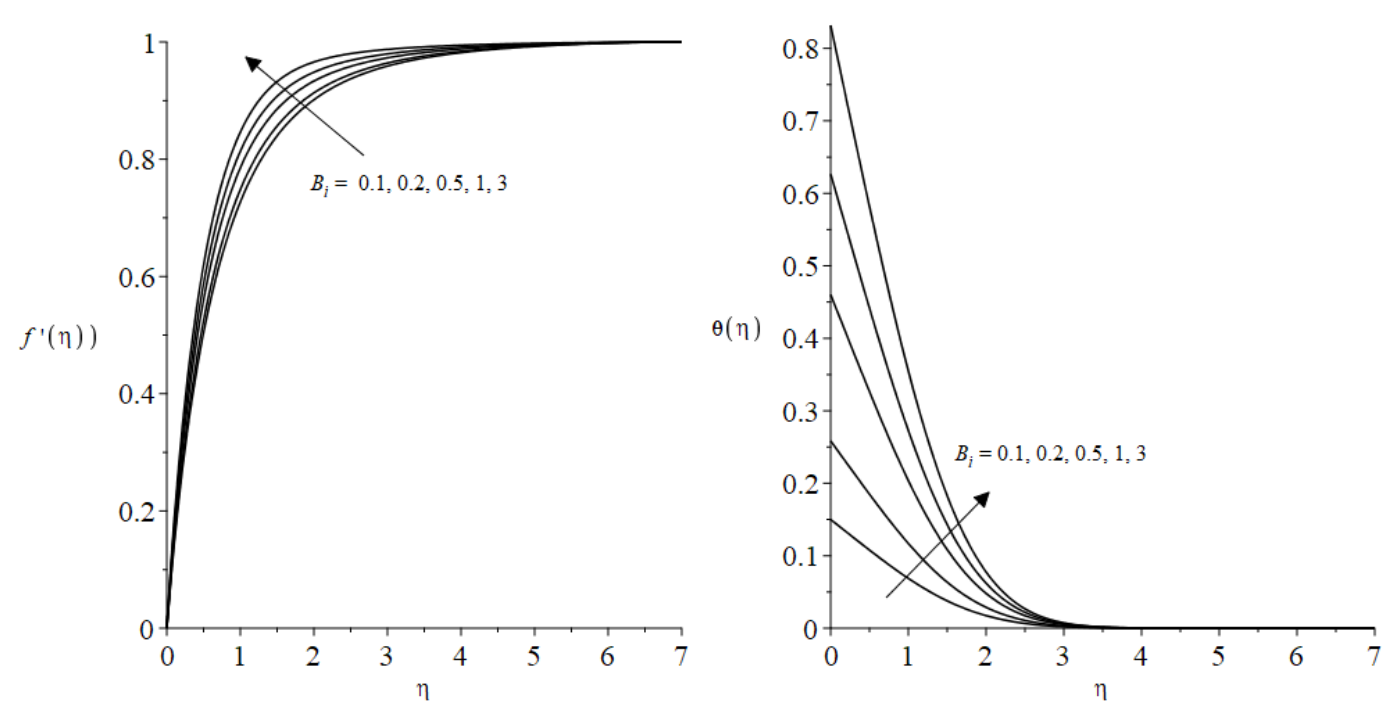

Fig. 4. Effect of $B i$ on $f^{\prime}(\eta)$ and $\theta(\eta)$ when $\Gamma=0.1, k_{1}=0.3$ and $\lambda=1$
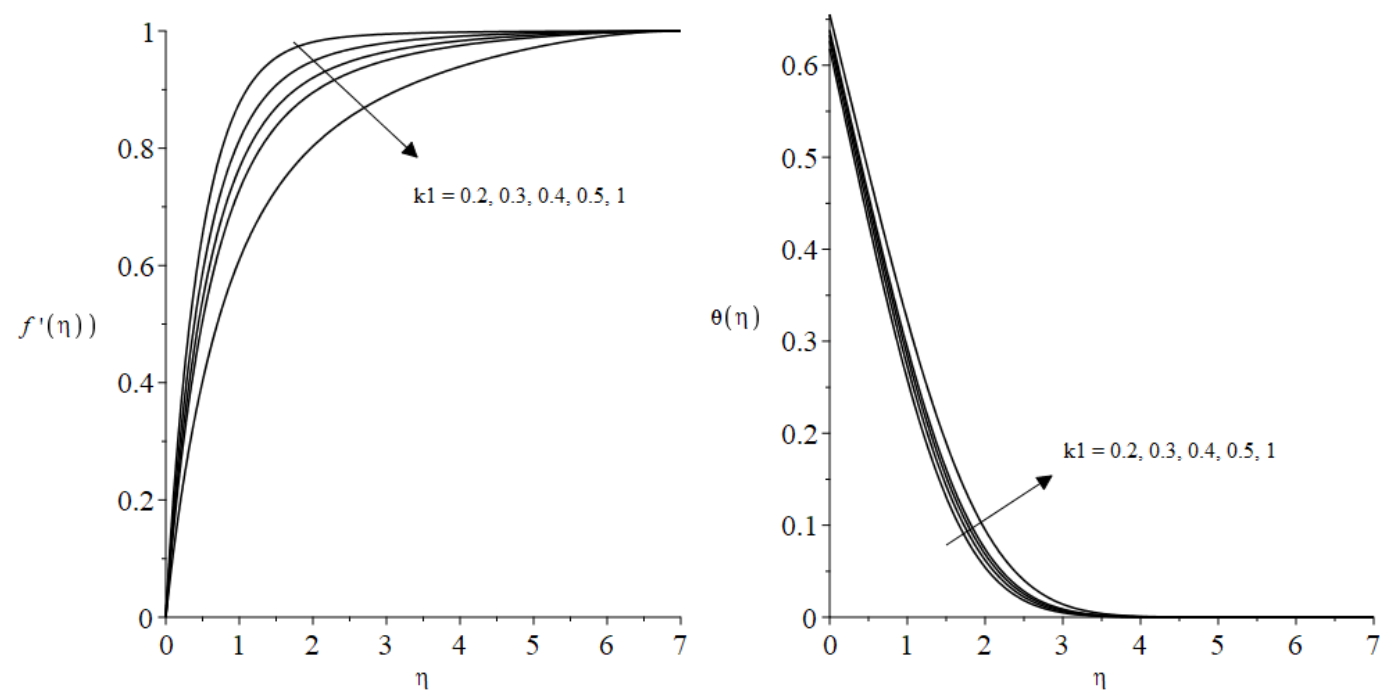

Fig. 5. Effect of $k_{1}$ on $f^{\prime}(\eta)$ and $\theta(\eta)$ when $\Gamma=0.1, \lambda=1$ and $B i=1$

\section{Conclusion}

The impact of pertinent parameters for combined convective transport of Brinkman-viscoelastic model over a horizontal circular cylinder saturated in porous region is studied. The parameters of $\Gamma$ , $k_{1}, B i$ and $\lambda$ are investigated on the flow features and heat transfer characteristics. To summarize, all of the parameters significantly affect the fluid flow behaviors. The impact of increasing the $\Gamma, k_{1}$ ,$B i$ and $\lambda$ is contradict on the velocity and temperature profiles. Theoretical results will aid researchers, particularly in the manufacturing industry, in validating experimental study data.

\section{Acknowledgement}

The authors wish to thank the Universiti Malaysia Pahang for its financial support of this project, which was provided by RDU210707 and PGRS 2003169. A special thanks also goes to Universiti Teknologi MARA Cawangan Kelantan for their assistance in financing this research fund (600CK(PJIA/URMI 5/1)). 


\section{References}

[1] Ashraf, Muhammad, and Zia Ullah. "Effects of variable density on oscillatory flow around a non-conducting horizontal circular cylinder." AIP Advances 10, no. 1 (2020): 015020. https://doi.org/10.1063/1.5127967

[2] Basha, H. Thameem, R. Sivaraj, V. Ramachandra Prasad, and O. Anwar Beg. "Entropy generation of tangent hyperbolic nanofluid flow over a circular cylinder in the presence of nonlinear Boussinesq approximation: a nonsimilar solution." Journal of Thermal Analysis and Calorimetry 143, no. 3 (2021): 2273-2289. https://doi.org/10.1007/s10973-020-09981-5

[3] Mahat, Rahimah, Noraihan Afiqah Rawi, Sharidan Shafie, and Abdul Rahman Mohd Kasim. "Mixed Convection Boundary Layer Flow of Viscoelastic Nanofluid Past a Horizontal Circular Cylinder with Convective Boundary Condition." International Journal of Mechanical Engineering and Robotics Research 8, no. 1 (2019): 87-91. https://doi.org/10.18178/ijmerr.8.1.87-91

[4] Swalmeh, M. Z., H. T. Alkasasbeh, A. Hussanan, and M. Mamat. "Numerical Study of Mixed Convection Heat Transfer in Methanol based Micropolar Nanofluid about a Horizontal Circular Cylinder." In Journal of Physics: Conference Series, vol. 1366, no. 1, p. 012003. IOP Publishing, 2019. https://doi.org/10.1088/1742$6596 / 1366 / 1 / 012003$

[5] Zokri, S. M., N. S. Arifin, M. K. A. Mohamed, M. Z. Salleh, A. R. M. Kasim, and N. F. Mohammad. "Numerical solution on mixed convection boundary layer flow past a horizontal circular cylinder in a jeffrey fluid with constant heat flux." In AIP Conference Proceedings, vol. 1870, no. 1, p. 040034. AIP Publishing LLC, 2017. https://doi.org/10.1063/1.4995866

[6] Zokri, Syazwani Mohd, Nur Syamilah Arifin, Muhammad Khairul Anuar Mohamed, Abdul Rahman Mohd Kasim, Nurul Farahain Mohammad, and Mohd Zuki Salleh. "Mathematical model of mixed convection boundary layer flow over a horizontal circular cylinder filled in a Jeffrey fluid with viscous dissipation effect." Sains Malaysiana 47, no. 7 (2018): 1607-1615. https://doi.org/10.17576/jsm-2018-4707-32

[7] Zokri, Syazwani Mohd, Nur Syamilah Arifin, Abdul Rahman Mohd Kasim, and Mohd Zuki Salleh. "Free Convection Boundary Layer Flow of Jeffrey Nanofluid on a Horizontal Circular Cylinder with Viscous Dissipation Effect." Journal of Advanced Research in Micro and Nano Engineering 1, no. 1 (2020): 1-14.

[8] Zokri, Syazwani Mohd, Nur Syamilah Arifin, Muhammad Khairul Anuar Mohamed, Mohd Zuki Salleh, Abdul Rahman Mohd Kasim, and Nurul Farahain Mohammad. "Influence of radiation and viscous dissipation on magnetohydrodynamic Jeffrey fluid over a stretching sheet with convective boundary conditions." Malaysian Journal of Fundamental and Applied Sciences 13, no. 3 (2017): 279-284.

[9] Kasim, Abdul Rahman Mohd, Nur Syamilah Arifin, Syazwani Mohd Zokri, and Mohd Zuki Salleh. "Fluid-particle interaction with buoyancy forces on Jeffrey fluid with Newtonian heating." CFD Letters 11, no. 1 (2019): 1-16.

[10] Kasim, Abdul Rahman Mohd, Nur Syamilah Arifin, Noor Amalina Nisa Ariffin, Mohd Zuki Salleh, and Muhammad Imran Anwar. "Mathematical model of simultaneous flow between Casson fluid and dust particle over a vertical stretching sheet." International Journal of Integrated Engineering 12, no. 3 (2020): 253-260.

[11] Arifin, Nur Syamilah, Syazwani Mohd Zokri, Abdul Rahman Mohd Kasim, Mohd Zuki Salleh, and Noor Amalina Nisa Arifin. "Jeffrey fluid embedded with dust particles over a shrinking sheet: A numerical investigation." Journal of Advanced Research in Fluid Mechanics and Thermal Sciences 74, no. 2 (2020): 196-209. https://doi.org/10.37934/arfmts.74.2.196209

[12] Tham, Leony, and Roslinda Nazar. "Numerical solution of mixed convection flow about a sphere in a porous medium saturated by a nanofluid: Brinkman model." Journal of Science and Technology 4, no. 2 (2012): 35-46.

[13] Tham, Leony, Roslinda Nazar, and loan Pop. "Mixed convection boundary layer flow past a horizontal circular cylinder embedded in a porous medium saturated by a nanofluid: Brinkman model." Journal of Porous Media 16, no. 5 (2013): 445-457. https://doi.org/10.1615/JPorMedia.v16.i5.50

[14] Ali, Farhad, Syed Aftab Alam Jan, Ilyas Khan, Madeha Gohar, and Nadeem Ahmad Sheikh. "Solutions with special functions for time fractional free convection flow of Brinkman-type fluid." The European Physical Journal Plus 131, no. 9 (2016): 1-13. https://doi.org/10.1140/epjp/i2016-16310-5

[15] Siyal, Ambreen, Kashif Ali Abro, and Muhammad Anwar Solangi. "Thermodynamics of magnetohydrodynamic Brinkman fluid in porous medium." Journal of Thermal Analysis and Calorimetry 136, no. 6 (2019): 2295-2304. https://doi.org/10.1007/s10973-018-7897-0

[16] Islam, Saeed, Muhammad Asif, and Samiul Haq. "Exact Solutions of Brinkman Type Fluid Between Side Walls Over an Infinite Plate." Preprints (2018): 2018020034.

[17] Kausar, Muhammad Salman, Abid Hussanan, Mustafa Mamat, and Babar Ahmad. "Boundary layer flow through Darcy-Brinkman porous medium in the presence of slip effects and porous dissipation." Symmetry 11, no. 5 (2019): 659. https://doi.org/10.3390/sym11050659

[18] Yadav, Dhananjay. "The onset of Darcy-Brinkman convection in a porous medium layer with vertical throughflow 
and variable gravity field effects." Heat Transfer 49, no. 5 (2020): 3161-3173. https://doi.org/10.1002/htj.21767

[19] Aziz, Laila Amera, Abdul Rahman Mohd Kasim, H. A. M. Al-Sharifi, Mohd Zuki Salleh, Nurul Farahain Mohammad, Sharidan Shafie, and Anati Ali. "Influence of aligned MHD on convective boundary layer flow of viscoelastic fluid." In AIP Conference Proceedings, vol. 1842, no. 1, p. 030005. AIP Publishing LLC, 2017. https://doi.org/10.1063/1.4982843

[20] Aziz, L. A., A. R. M. Kasim, and M. Z. Salleh. "Development on mathematical model of convective boundary layer flow of viscoelastic fluid with microrotation effect under constant wall temperature thermal condition over a bluff body." ASM Science Journal 12 (2019): 86-90.

[21] Mishra, S. R., R. S. Tripathy, and G. C. Dash. "MHD viscoelastic fluid flow through porous medium over a stretching sheet in the presence of non-uniform heat source/sink." Rendiconti del Circolo Matematico di Palermo Series 2 67, no. 1 (2018): 129-143. https://doi.org/10.1007/s12215-017-0300-3

[22] Mahat, Rahimah, Noraihan Afiqah Rawi, Abdul Rahman Mohd Kasim, and Sharidan Shafie. "Mixed convection flow of viscoelastic nanofluid past a horizontal circular cylinder with viscous dissipation." Sains Malaysiana 47, no. 7 (2018): 1617-1623. https://doi.org/10.17576/ism-2018-4707-33

[23] Mahat, Rahimah, Noraihan Afiqah Rawi, Abdul Rahman Mohd Kasim, and Sharidan Shafie. "Heat generation effect on mixed convection flow of viscoelastic nanofluid: convective boundary condition solution." Malaysian Journal of Fundamental and Applied Sciences 16, no. 2 (2020): 166-172. https://doi.org/10.11113/mjfas.v16n2.1367

[24] Wahid, Nur Syahirah, Mohd Ezad Hafidz Hafidzuddin, Norihan Md Arifin, Mustafa Turkyilmazoglu, and Nor Aliza Abd Rahmin. "Magnetohydrodynamic (MHD) Slip Darcy Flow of Viscoelastic Fluid Over A Stretching Sheet and Heat Transfer with Thermal Radiation and Viscous Dissipation." CFD Letters 12, no. 1 (2020): 1-12.

[25] Al-Sharifi, H. A. M., A. R. M. Kasim, M. Z. Salleh, N. Md Sarif, N. F. Mohammad, S. Shafie, and A. Ali. "Influence of slip velocity on convective boundary layer flow of Jeffrey fluid under convective boundary conditions." ARPN Journal of Engineering and Applied Sciences 11, no. 18 (2006): 10950-10953.

[26] Ashraf, M. Bilal, T. Hayat, S. A. Shehzad, and A. Alsaedi. "Mixed convection radiative flow of three dimensional Maxwell fluid over an inclined stretching sheet in presence of thermophoresis and convective condition." AIP Advances 5, no. 2 (2015): 027134. https://doi.org/10.1063/1.4913719

[27] Gaffar, S. Abdul, V. Ramachandra Prasad, and E. Keshava Reddy. "Magnetohydrodynamic free convection flow and heat transfer of non-Newtonian tangent hyperbolic fluid from horizontal circular cylinder with Biot number effects." International Journal of Applied and Computational Mathematics 3, no. 2 (2017): 721-743. https://doi.org/10.1007/s40819-015-0130-y

[28] Sarif, Norhafizah Md, Mohd Zuki Salleh, and Roslinda Nazar. "Mixed convection flow over a horizontal circular cylinder in a viscous fluid at the lower stagnation point with convective boundary conditions." ScienceAsia 42 (2016): 5-10. https://doi.org/10.2306/scienceasia1513-1874.2016.42S.005

[29] Sarif, Norhafizah Md, Sallhe Mohd Zuki, and Roslinda Nazar. "Mixed convection over a horizontal circular cylinder embedded in porous medium immersed in a nanofluid with convective boundary conditions at lower stagnation point: A numerical solution." In MATEC Web of Conferences, vol. 189, p. 02004. EDP Sciences, 2018. https://doi.org/10.1051/matecconf/201818902004

[30] Nazar, Roslinda, Norsarahaida Amin, Diana Filip, and Ioan Pop. "The Brinkman model for the mixed convection boundary layer flow past a horizontal circular cylinder in a porous medium." International Journal of Heat and Mass Transfer 46, no. 17 (2003): 3167-3178. https://doi.org/10.1016/S0017-9310(03)00122-4 